\title{
Robot Publicitario Aplicado A La Pedagogía Infantil
}

\section{Gladys Urquizo Buenaño}

Extensión Norte Amazónica, Escuela Superior Politécnica de Chimborazo,

Francisco de Orellana, Ecuador

Javier Gavilanes

Isabel Vaca

Leonela Villagómez

Facultad de Mecánica, Escuela Superior Politécnica de Chimborazo,

Riobamba, Ecuador

Angel Llerena

UNIR/Universidad Internacional de la Rioja, España

Doi:10.19044/esj.2018.v14n22p136 URL:http://dx.doi.org/10.19044/esj.2018.v14n22p136

\begin{abstract}
This paper focuses on presenting the design and build up of an interactive didactic robot. Its structure has an omnidirectional traction with WIFI communication for sending and receiving information between the user and the robot. Its structure is made up of two screens: one on the top of the robot, simulating a head, and another one on the front of its figure. It has a camera to obtain "vision" and proximity sensors to create a symbiosis relationship with the user. To control the devices, a Raspberry Pi 3 and an Arduino 328 card were used under a master slave system. The structure of the robot was designed using a 3D modeling, and the parts were created using a 3D printing. It has the following functions: manual control, tell a story, dance, advertising or game modes that can be selected from the user interface. This prototype places an emphasis on presenting applications that stimulate learning and logical understanding in children, leaving the possibility of being reprogrammed to add functions.
\end{abstract}

Keywords: Mobile Robots and Intelligent Autonomous Vehicles, Robot Programming and Multi-Robot Systems, Assistive, Service and Social Robotics

\section{Resumen}

Se presenta el diseño y construcción de un robot didáctico interactivo cuya estructura posee tracción omnidireccional con comunicación WIFI para el envío-recepción de información entre el usuario y el robot. Su estructura 
está conformada por dos pantallas, una en la parte superior del robot, simulando la cabeza y otra en la parte frontal del cuerpo; dispone de una cámara para obtener "visión" y sensores de proximidad para crear una relación de simbiosis con el usuario. Para controlar los dispositivos se emplearon una Raspberry Pi 3 y una tarjeta Arduino 328 bajo un sistema maestro esclavo. La estructura del robot fue diseñada mediante modelamiento 3D y las partes fueron creadas mediante impresión 3D. Tiene las siguientes funciones: control manual, relatar un cuento, modos baile, publicitario o juego que pueden ser seleccionadas desde la interfaz de usuario. El prototipo tiene énfasis en presentar aplicaciones que estimulen aprendizaje y comprensión lógica en los niños, dejando la posibilidad de ser reprogramado para añadir funciones.

Palabras clave: Robots móviles y vehículos autónomos inteligentes, Programación de robots y sistemas multi-robot, Robótica asistencial, de servicio y social

\section{Introducción}

Uno de los principales retos en el mundo de la robótica es la perfecta inmersión de robots en el entorno humano, para lo cual las investigaciones sobre la interacción entre robots y humanos son cada vez mayores, presentando cada vez mejores resultados, tales como la presencia de humanoides en diferentes escenarios como los robots meseros en restaurantes de Beijin, (Jyh-Hwa, L., \& Kuo, 2008) hasta los mejores hospitales del mundo, un ejemplo de esto es PEEPER.

Una de las áreas de la robótica es la robótica de servicios, la misma que presenta gran diversidad en los campos que cubre, tales como la asistencia personal, la limpieza, la vigilancia, la educación, la publicidad. Para catalogar a un robot como un robot de servicio se considera que su propósito es presentar funciones de servicio útiles orientadas al beneficio de la humanidad y que su modo de operación responda a una autonomía total o parcial. (Aracil, Balaguer, \& Armada, 2008).

Por su lado los robots didácticos buscan explícitamente la interacción con el ser humano en busca de desarrollar habilidades de kinestesia y viso espaciales mediante el uso de didáctica para potenciar competencias y habilidades relacionadas al pensamiento abstracto y motricidad gruesa y fina (Ibarra Quevedo \& Arteaga Bouchan, 2007).

El Ecuador actualmente empieza a dar apertura a investigaciones de este tipo con la finalidad de presentar soluciones innovadoras a empresas que pretenden llegar al usuario con la finalidad de entregarle información de manera actualizada. El marco nacional del Ecuador pretende estimular la investigación científica y tecnológica con el fin de una constante mejora en la 
productividad, respondiendo a las necesidades actuales y futuras del sector productivo (Acosta, 2010).

La presente investigación se centra en la implementación de un robot publicitario. Uno de los aspectos más importantes en la creación de un robot es el desplazamiento, la movilidad de una plataforma está directamente relacionada con la configuración de llantas que usa la plataforma móvil que sirve como base para el robot. En ésta área existen diferentes configuraciones desarrolladas, siendo las principales la de tipo diferencial, que es una configuración relativamente fácil tanto en implementación como en programación cuyo principio de funcionamiento es el suministro de voltaje a dos ruedas unidas a un mismo centro de eje, permitiendo un desplazamiento en línea recta y giros, pero que al estar compuesto únicamente por dos ruedas necesita de al menos una tercera para lograr una estabilidad; la configuración Ackerman está compuesta por dos ruedas delanteras que proporcionan dirección a la plataforma y dos ruedas traseras de tracción, el inconveniente de esta configuración es que su restricción no olonómica, por su parte la configuración de tipo triciclo es similar a la Ackerman en la cual la rueda delantera es la que le provee de dirección y tracción a la plataforma. La configuración Síncrona está compuesta por tres ruedas unidas entre sí, las ruedas proveen dirección y motricidad, y se mueven simultáneamente para lograr el desplazamiento deseado, su programación es de mayor complejidad a las anteriores (Sanz, 2011).

Posterior a estas configuraciones aparece el desplazamiento tipo omnidireccional que permite que la plataforma se desplace con mayor libertad permitiendo realizar movimientos en cualquier dirección, gracias a su característica de poder girar sin antes tener que desplazarse para orientarse. La principal consideración en la elección de la configuración y el tipo de llantas para una plataforma móvil es el entorno en el que se requiere que la plataforma se desplace, para realizar sus funciones objetivo, (Silva Ortigoza, Molina Vilchis, Hernández Guzmán, \& Silva Ortigoza, 2007).

Existen otras configuraciones de llantas que permiten que las plataformas se desplacen en direcciones específicas, cubriendo trayectorias simples o complejas, dependiendo de la posición de las llantas o de los ejes que las unen, conforme a cada caso, así como también la variedad en los tipos de llantas cada vez es mayor.

Por su parte la introducción de robots en la pedagogía muestra cambios significativos en el interés de los estudiantes (Romero, 2012), lo que se refleja en el aprendizaje obtenido con la intervención de dichos dispositivos, un estudio realizado en la ciudad de Bogotá en el año 2014 en poblaciones escolares de instituciones públicas comprendidas entre los 9 a los 15 años de edad, revela cifras que llegan hasta un $72,5 \%$ presentaron mejorías en el aprendizaje de los estudiantes mientras que el 27,5 no presentaron mejorías. 
La investigación fue de tipo descriptivo y exploratorio con una población mayor a 2000 estudiantes (Pedraza, 2014).

En el presente trabajo se diseñó y construyó un robot publicitario orientado a la pedagogía infantil, con el fin de insertar este dispositivo en los procesos de aprendizaje al evidenciar la respuesta que recibe la invención por parte del usuario en la interacción con dispositivos interactivos.

\section{Materiales y métodos}

El sistema está compuesto por un sistema de locomoción o desplazamiento, un sistema de potencia para proporcionarle torque a la plataforma, un sistema de control para el envío de señales eléctricas a los dispositivos electrónicos, y una estructura física que aloje todos los componentes, son los elementos generales necesarios para implementar el robot. El sistema electrónico está conformado por una tarjeta Raspberry PI 3 Master y un Arduino 328 junto a una segunda Raspberry PI3 conectados como esclavos (Wheat, 2012). El master controla directamente a una pantalla de 7 pulgadas oficial Raspberry, una cámara Raspberry pi, un parlante, los cables de comunicación i2c y una batería de $2 \mathrm{~A}$. La segunda Raspberry tiene control sobre la pantalla de 5 pulgadas oficial de Raspberry y una batería de 2 A, mientras que el Arduino 328, se encarga de la parte de potencia de la plataforma, está conectada a una tarjeta de expansión arduino para tener el control de 2 servomotores (Vega \& López, 2006), 3 motores con encoder, 3 llantas omnidireccionales de $48 \mathrm{~mm}, 3$ sensores ultrasónicos dual (dus), una batería de 12 v NI-MH, y los cables para la comunicación i2c, como se detalla en la Figura 1.
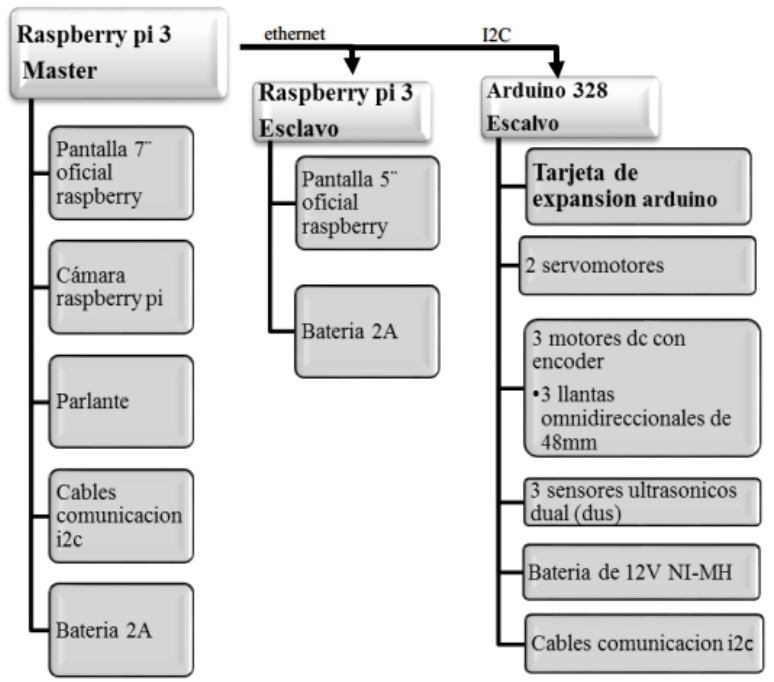

Figura 1. Conexión de Dispositivos Electrónicos Fuente: Vaca I, Villagómez L. 


\section{Raspberry PI 3}

Un computador de tamaño reducido, presenta entre sus principales características un procesador central de 1,2 GHZ, 1 GB de RAM, Wireless, Bluetooth, puertos USB, puerto Ethernet, interfaz de cámara CSI, interfaz de pantalla, entre otras.

La adquisición de información visual del entorno hacia el robot se la realizó a través de imágenes obtenidas por una cámara, que se integra mediante un módulo disponible para Raspberry, que permite grabar videos de alta definición en 3 modos: 1080 p 30, 720 p 60 y VGA 90 y también fotografías de alta calidad; se logra mediante conexión al puerto CSI de la Raspberry.

Para conectar la pantalla oficial Raspberry PI se usó un cable de cinta al puerto DSI, un conversor de potencia y de señal, y dos conexiones, la primera que es para la alimentación de energía hacia el puerto GPIO de la Pi y la segunda que sirve de conexión al puerto DSI presente en la raspberry, permite hasta 10 dedo- táctil, capacitiva, evitando la necesidad de un teclado o un ratón; funciona a través del sistema operativo de Raspbian. Las dimensiones de la pantalla de 7 pulgadas son $155 \mathrm{~mm}$ x $86 \mathrm{~mm}$ de pantalla visible al usuario, mientras que la pantalla de 5 pulgadas es de $109 \mathrm{~mm} \times$ $76 \mathrm{~mm}$, tiene una resolución de 800 x 480 pixeles (Raspberry, 2016).

\section{Arduino ATmega 328}

El arduino ATmega 328 puede operar en diferentes gamas de voltaje, entregando mayor velocidad de reloj en el procesador a medida que se incremente la tensión de alimentación a la placa, ejemplo de esto es la velocidad de reloj que llega hasta $4 \mathrm{MHZ}$ con una alimentación de placa de $1.8 \mathrm{~V}$, mientras que alcanza una velocidad de $20 \mathrm{Mhz}$ con una alimentación de placa de al menos 4,5 V. La placa de expansión IO Arduino permite conexión mediante XBee Pro, tiene unas salidas que le permiten establecer comunicación mediante una conexión RS485, tiene pines de salida para PWM(modulador de ancho de pulso) y es compatible con protocolos de comunicación IIC, I2C y TWI., sus características físicas son ideales para integrarlo discreta y eficientemente en la placa de soporte, adicional a éstas características la tarjeta arduino ATmega 328 tiene un lenguaje de programación de tipo $\mathrm{C}++$, de fácil manejo con diferentes librerías desarrolladas para diferentes aplicaciones y que permiten una interactividad eficiente entre las señales de entrada de los diversos sensores y la tarjeta; la Figura 2 muestra el tipo de comunicación entre las tarjetas (Diaz, 2016). 


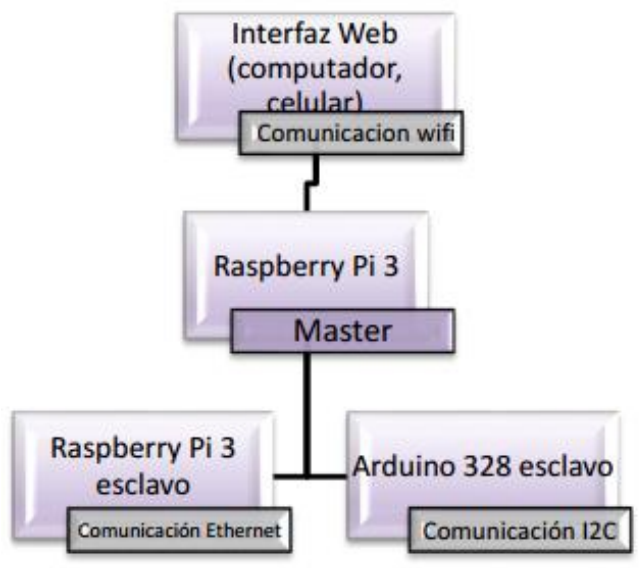

Figura 2. Sistema de Comunicación del Robo Fuente: Vaca I, Villagómez L.

\section{Sistemas de Potencia, sensores y estructura}

La detección de aproximación del usuario hacia el robot, se realiza mediante un sensor ultrasónico conectado al arduino a través de una conexión RS485, la tensión necesaria para su funcionamiento de dicho sensor es de $5 \mathrm{v}$, presenta un consumo de $20 \mathrm{~mA}$, el rango de distancia de funcionamiento, o distancia de alcance de medición del sensor, es de 4 a $300 \mathrm{~cm}$, con una frecuencia de $40 \mathrm{kHz}$. Para impulsar el movimiento del robot se acoplaron dos motores de corriente continua a la plataforma, las señales de pulso del encoder, mide el ángulo de rotación del motor, la señal es retroalimentada y usada para calcular la velocidad del motor, apoyándose en cajas de engranajes para aumentar el torque de salida. Una batería NI-MH abastece los requerimientos de tensión de los dispositivos electrónicos, ésta batería usa una aleación de hidruro metálico y de hidróxido de níquel, y está formada por pequeñas celdas que arrojan 1,2 V cada una. Para el diseño de la estructura, se empleó la herramienta Solidworks, software que permite crear el diseño de piezas con precisión milimétrica (Planchard, 2015) y que tiene la ventaja de exportar los proyectos creados en archivos de código g, el mismo que es el archivo que se usa como entrada de programación para las máquinas de control numérico computarizado (Cortés Cherta, 1995). La Figura 3 muestra el diseño de la cabeza del robot. 


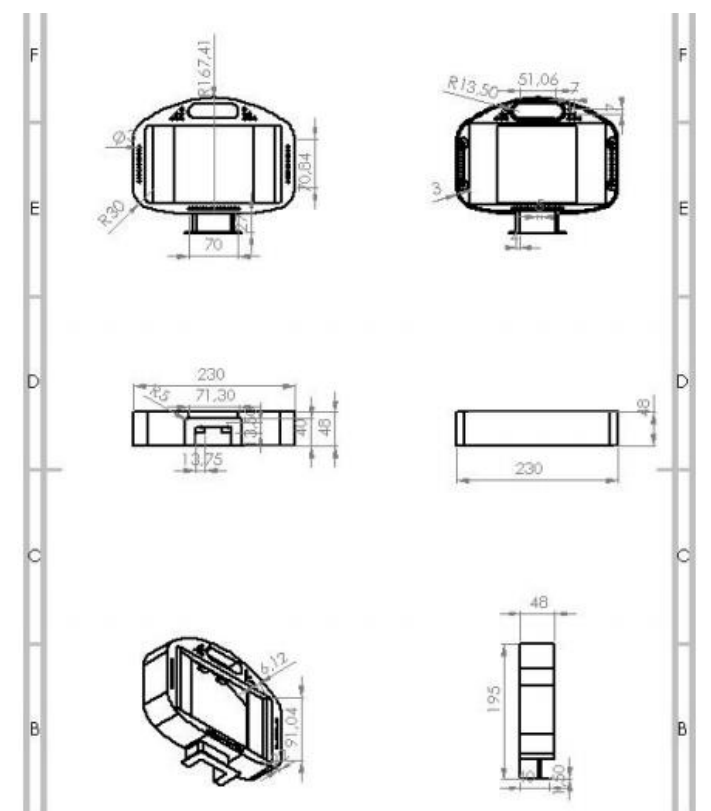

Figura 3. Diseño de Piezas del Robot Fuente: Vaca I, Villagómez L.

Por su parte la estructura física del Robot se muestra en la figura 4, la misma que tiene una base diseñada con modelación CAD e impresa en PLA a través de impresión 3D que permite realizar movimientos en todas las direcciones mediante 3 llantas omnidireccionales, la base también aloja las baterías de alimentación de todo el robot. En un segundo nivel se alojan la tarjeta Arduino 328, los sensores de proximidad y la tarjeta de expansión Arduino I/O. La combinación del accionar de los motores permiten movimientos del plataforma hacia adelante, hacia atrás, hacia la derecha, hacia la izquierda, giro a la derecha y giro hacia la izquierda.
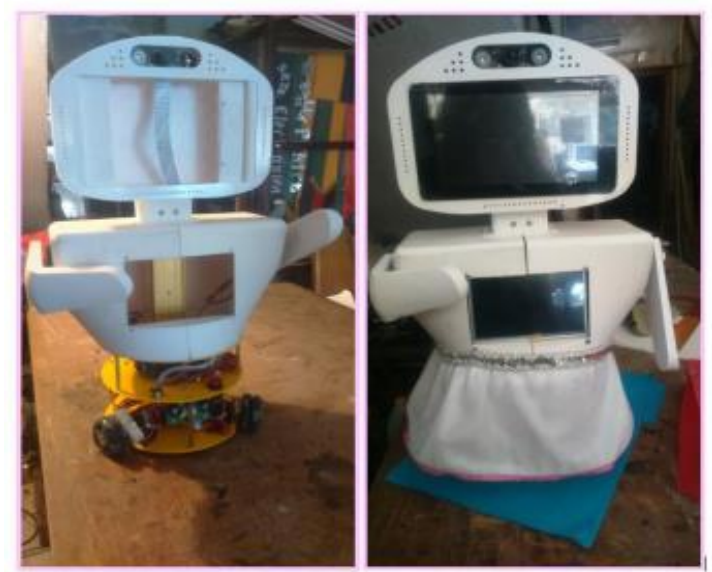

Figura 4. Robot ensamblado en su parte frontal y posterior, respectivamente. Fuente: Vaca I, Villagómez L. 
La plataforma tiene articuladas a la base 3 ruedas de tipo omnidireccional, ubicadas simétricamente en lugares estratégicos, lo que le proporciona 3 grados de libertad al robot, eliminando en gran parte de limitaciones cinemáticas. La Figura 5 indica los posibles giros de cada una de las ruedas, esta configuración de ruedas de tipo omnidireccional le brinda al robot la posibilidad de desplazarse en todas las direcciones, lo que se logra con la programación en la alimentación a los motores M1, M2, y M3, cada uno correspondiente a una rueda, que hacen que las ruedas giren, tal como se indica en la Tabla 1.

\begin{tabular}{|c|c|c|c|}
\hline Desplazamiento & Motor M1 & Motor M2 & Motor M3 \\
\hline Adelante & OFF & ON & ON \\
\hline Atrás & OFF & ON & ON \\
\hline Izquierda & ON & ON & ON \\
\hline Derecha & ON & ON & ON \\
\hline Giro Derecha & ON & ON & ON \\
\hline Giro Izquierda & ON & ON & ON \\
\hline
\end{tabular}

Tabla 1. Accionamiento de los motores dependiendo del movimiento deseado en las ruedas.

Al encontrarse articuladas las ruedas a la plataforma en un punto de contacto, y al alimentar voltaje a los motores que permiten que se giren se logra un giro perpendicular al centro de giro de cada rueda con la plataforma, y el sentido de giro depende del voltaje que se les inyecte, mismo que puede ser positivo o negativo (Cortés Cherta, 1995). Para lograr que la plataforma se desplace hacia adelante es necesario inyectarle un voltaje positivo al motor M3, correspondiente a la rueda frontal lateral derecha, lo que permite que gire en sentido favorable al giro de las manecillas del reloj, mientas que es necesario alimentación de voltaje negativo al motor M2 correspondiente a la rueda frontal lateral izquierda, lo que permite que gire en sentido contrario al giro de las manecillas del reloj, por su parte el motor M3 se mantiene apagado, permitiendo que la rueda posterior del robot se deslice sin oponerse al movimiento debido a su característica de omnidireccionalidad.

Para el desplazamiento hacia atrás de la plataforma se debe alimentar de voltaje negativo al motor M3 logrando que gire en sentido contrario al sentido de las manecillas del reloj, por su parte el motor M2 debe recibir alimentación positiva logrando un giro con sentido igual al giro de las manecillas del reloj, y de la misma forma que el caso anterior el motor de la rueda posterior se mantiene apagado lo que permite que la plataforma se desplace en este caso hacia atrás. Para el desplazamiento a la izquierda se requiere un valor de voltaje negativo al motor $\mathrm{M} 3$, correspondiente a la rueda frontal lateral derecha, lo que permite que gire en sentido contrario al giro de las manecillas del reloj, un voltaje negativo al motor M2 correspondiente a la rueda frontal lateral izquierda, lo que permite que gire en sentido contrario al giro de las manecillas del reloj, y un voltaje positivo al motor M3 
correspondiente a la rueda posterior de la plataforma, lo que permite que gire en sentido favorable al giro de las manecillas del reloj.

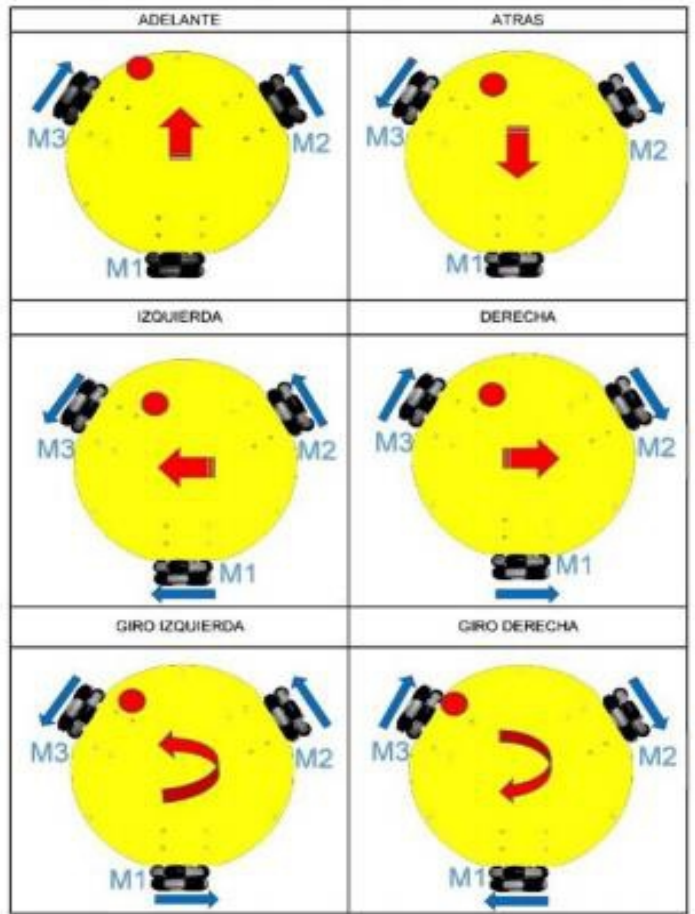

Figura 5. Movimientos de las llantas de la plataforma, con sus motores M1, M2, M3 correspondientemente.

Para el desplazamiento a la derecha se requiere un valor de voltaje positivo al motor M3, lo que permite que gire en sentido favorable al giro de las manecillas del reloj, un voltaje positivo al motor M2 lo que permite que gire en sentido igual al giro de las manecillas del reloj, y un voltaje negativo al motor M3, haciéndolo girar en sentido contrario al giro de las manecillas del reloj. El giro hacia el lado izquierdo se requiere un valor de voltaje negativo al motor $\mathrm{M} 3$, lo que permite que gire en sentido contrario al giro de las manecillas del reloj, un voltaje negativo al motor M2 voltaje que produce en la rueda y un giro en sentido contrario al giro de las manecillas del reloj, y un voltaje negativo al motor $\mathrm{M} 3$, lo que permite que la rueda de atrás gire en sentido favorable al giro de las manecillas del reloj.

Finalmente para el giro de la plataforma a la derecha se requiere un valor de voltaje positivo al motor M3, lo que permite que gire en sentido favorable al giro de las manecillas del reloj, un voltaje positivo al motor M2 lo que permite que gire en sentido igual al giro de las manecillas del reloj, y un voltaje positivo al motor M3, haciéndolo girar en sentido favorable al giro de las manecillas del reloj. 


\section{Comunicación}

Para las conexiones que permiten la comunicación entre los dispositivos electrónicos así como para la comunicación i2c se empleó cable de cinta DSI. El sistema implementado en el robot cuenta con una interfaz Web que permite una reprogramación por bloques del robot (Somolinos Sánchez, 2002), para lo cual es necesario acceder a la red wifi del robot mediante una dirección IP específica, mientras que la comunicación entre la Raspberry Pi que hace las funciones de master y la Raspberry que trabaja como esclavo funciona mediante comunicación Ethernet (González, 2002).

Por su parte la comunicación entre el máster y el Arduino que trabaja como esclavo se lo realiza con comunicación síncrona $\mathrm{I} 2 \mathrm{C}$ que permite velocidades de hasta 2.4 Mbits/sg; para lo cual se necesitan dos conexiones, la de reloj y la de envío-recepción de datos entre el master y el esclavo, tal como se indica en la Figura 6 (Cortés Cherta, 1995).

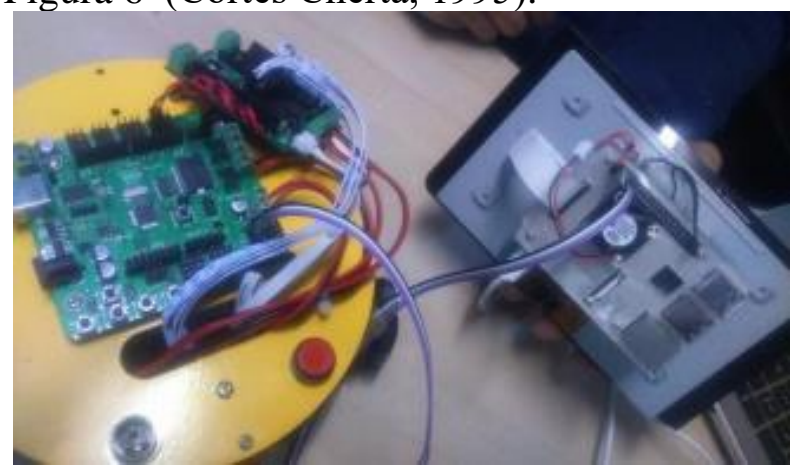

Figura 6. Conexión Raspberry - Arduino Fuente: Vaca I, Villagómez L.

\section{Programación del Robot}

La programación del robot está desarrollada mediante nodos de programación en PYTHON, Figura 7, un lenguaje de programación interpretado y orientado a objetos; es de acceso libre y continuamente brinda mejores prestaciones al usuario debido a que tiene un modelo de desarrollo comunitario (Zabala, 2007).

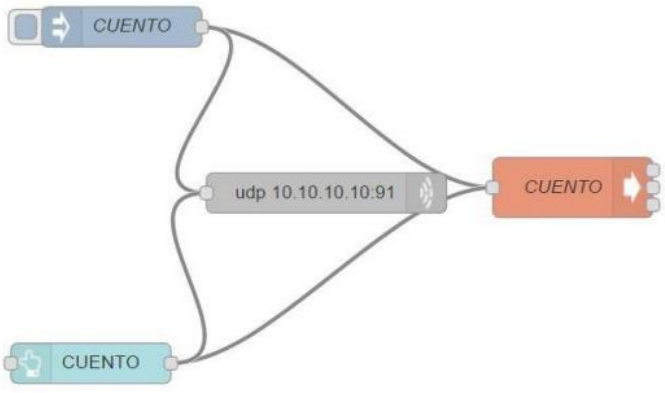

Figura 7. Nodos de direccionamiento en la programación Fuente: Vaca I, Villagómez L. 
El diagrama de flujo de la programación del robot Figura 8, tiene como inicio la pregunta del modo en el cual se encuentra el robot, la primera opción es MODO MANUAL, el programa pregunta si la información que ha elegido el usuario corresponden a la función modo manual en caso afirmativo los sensores se activan, el programa lee las variables de entrada que corresponden a los sensores para interpretar la información recibida y envía valores en la variables de salida que corresponden a la base, estos valores llevan la información necesaria de accionamiento hacia los motores de las llantas para que el robot se mueva hacia adelante, atrás, izquierda, derecha o que gire hacia la derecha o la izquierda; así como también llevan información hacia la pantalla ubicada en la parte superior o cabeza, la misma que por su parte puede indicar imágenes correspondientes a las figuras prediseñadas y alojadas en la memoria del robot, que representan una cara seria, cara enojada, cara normal, guiño, busca o cara sorprendida. Siguiendo el diagrama de flujo la segunda opción sobre el modo de selección del robot el programa pregunta si el posicionamiento del modo del robot se encuentra en modo baile, en caso afirmativo los sensores se apagan y el programa no recibe valores en las variables de entrada que corresponden a los sensores, y envía información, que corresponden a señales de accionamiento, a través de las variables de salida de la base de la plataforma. La interpretación de esta información se traduce como el accionamiento de los motores de las llantas que le permiten a la plataforma realizar una serie de instrucciones, cuya ejecución se convierte en una secuencia de baile programada, o sea movimientos fijos preestablecidos; dentro de este modo la siguiente instrucción del programa es el envío de información hacia la pantalla superior para mostrarle al usuario expresiones predefinidas, con el fin de evidenciar los movimientos simulados ante el usuario, todo esto con información de salida que corresponde a la música que se reproduce mediante los parlantes del robot; si ésta no es la opción seleccionada entonces el programa hace la tercera pregunta que busca saber si el usuario seleccionó la opción cuento, si esta opción es verdadera, los sensores se apagan, el programa envía datos de salida de información que se traduce en una serie de imágenes que se muestran en la pantalla de la parte superior del robot expresiones predefinidas.

Las imágenes corresponden a la simulación de movimientos en los labios, para simular la gesticulación en los labios que se produce al pronunciar palabras, en este caso para crear en ambiente de relato del cuento, a su vez se envía también información que se muestra en la pantalla inferior que se traduce en la reproducción de la letra del cuento que se está relatando a través de los parlantes, si ésta tampoco es la opción seleccionada, el programa pregunta la quinta opción que corresponde al modo juego, si es la opción seleccionada los sensores se apagan, y el programa envía información que se muestra en las pantallas superiores e inferiores como expresiones prefijadas, 
en caso de ser negativa esta opción el programa realiza la pregunta sí está en modo publicidad, sí es correcto, los sensores se apagan, la pantalla superior indica una serie de expresiones que simulan la vocalización de palabras, mientras que en la pantalla inferior se muestra la información en forma de letras que contienen la publicidad orientada al público y se envía información que reproduce un audio con la misma información, en caso de ser negativo la elección de la función, se procede a la última opción que es Twitter, la cual si es seleccionada por el usuario el programa envía información que apaga los sensores, y activa la opción foto carnet, que consiste en adquirir una foto con la cámara implementada en la parte frontal del robot, y tratada en el programa para su posterior envío hacia la red a través de la aplicación twitter.

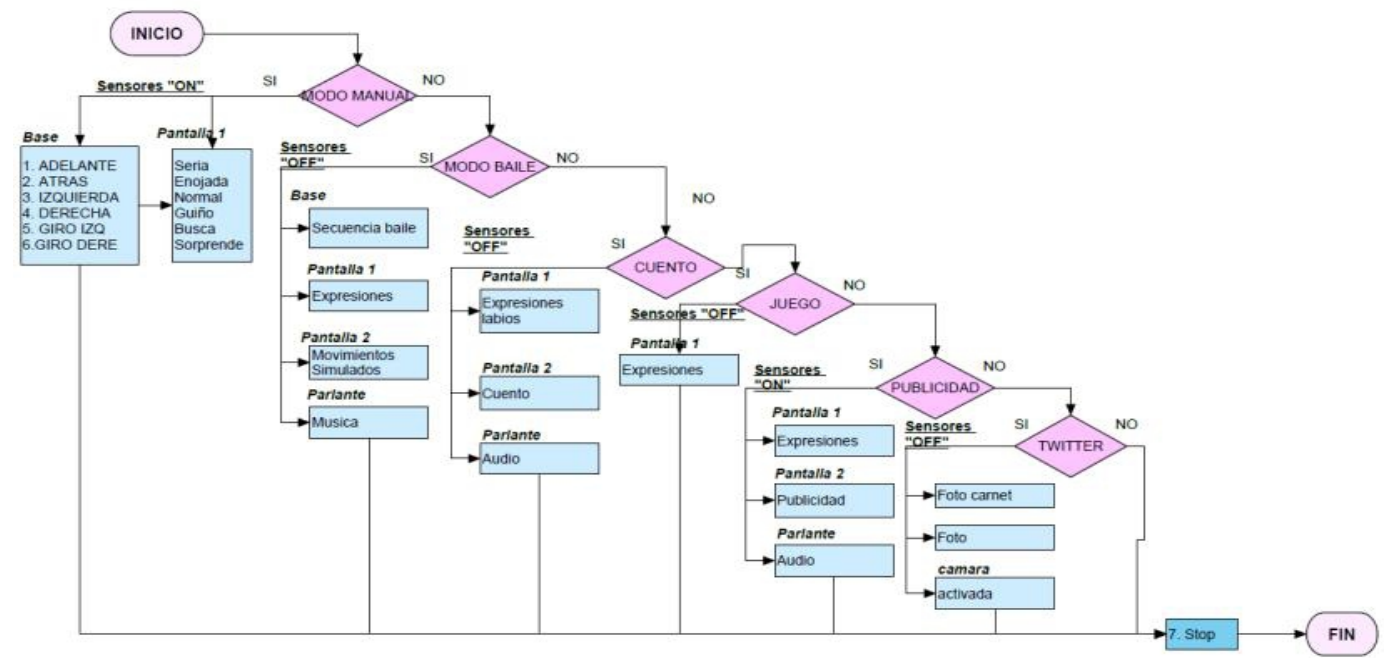

Figura 8. Diagrama de flujo del funcionamiento del robot. Fuente: Vaca I, Villagómez L.

\section{Resultados y Discusiones}

Modos de Funcionamiento del Robot. La Figura 9 indica las funciones del robot, la primera hace referencia a la función CONTROL MANUAL del robot le permite al usuario tener control sobre los movimientos de las llantas y los brazos mediante comandos, que son ingresados al GPIO y direccionados a través del switch del código del arduino hacia los actuadores correspondientes para su ejecución, el ingreso de información del usuario se lo realiza mediante la interfaz de la pantalla inferior que permite elegir entre las opciones de funcionamiento, para lo cual se generó un dashboard web, mediante bloques generados con Node-Red, que adquieren información de la pantalla táctil al tener contacto sobre algún punto de su superficie, tales como el comando 1 que envía la información adecuada para que la plataforma avance hacia adelante, el 2 para que retroceda, el 3 para STOP, 4 para avanzar 
a la derecha, 5 izquierda, 6 rotar a la izquierda, 7 rotar a la derecha, 8 brazo derecha arriba, y otros.

Por su parte la Raspberry pi también puede recibir comandos ingresados por el usuario, el comando 2 hace que muestre la imagen que corresponde a "una cara" para "Busca", 3 guiño. 5 pestañeo, entre otros.

El modo autónomo del robot depende de la selección del funcionamiento que elija el usuario, las cuales son: el MODO BAILE, el Robot realiza movimientos de forma sincronizada con la música que reproduce en ésta función.

La función cuento que está programada en el robot reproduce un audio de cuentos grabados en la memoria, al mismo tiempo que muestra en la pantalla superior diferentes imágenes que simulan el movimiento de labios al relatar un CUENTO, mientras que en la pantalla inferior se indica el cuento para que pueda ser leído por el usuario.

La función de JUEGO del robot le permite interactuar con el usuario, en este modo de funcionamiento se envían órdenes para ejecutar un script, que activa la opción de vocabulario, ésta opción permite la enseñanza de palabras tanto en quechua como en inglés.

La función programada para PUBLICIDAD trasmite información seleccionada y previamente almacenada en la memoria interna, en esta opción el robot activa su salida de audio mediante los parlantes implementados para este fin y sus pantallas para la visualización de dicha información.

La función de redes sociales Twitter-Facebook, presenta dos opciones para los usuarios, la primera que consiste en adquirir una imagen desde la cámara instalada en el robot y mediante un procesamiento de imágenes crear un carnet con la información adquirida para luego ser presentada en la pantalla superior del robot con los detalles programados, y la segunda que consiste en tomar una fotografía del usuario y publicarla directamente en las páginas de las redes sociales creadas para publicar la información adquirida por el robot, explícitamente las imágenes seleccionadas en caso de ser requeridas por el usuario.

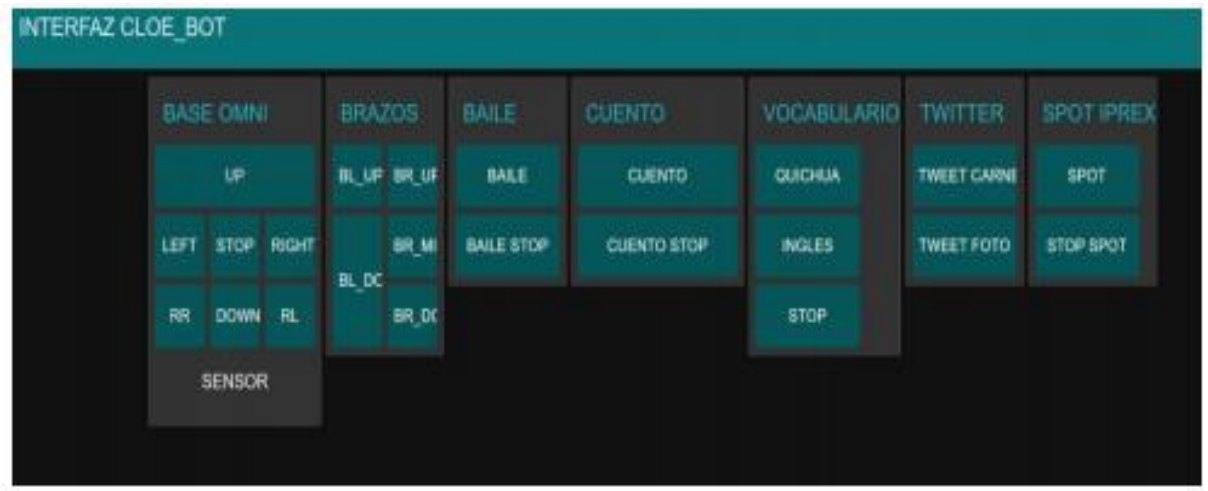

Figura 9. Funciones del Robot 


\section{Influencia en la Pedagogía Infantil}

Para transmitir información como parte de la educación infantil el empleo de un dispositivo amigable a su entorno es un medio alternativo, debido al interés que crean en los infantes gracias a la apariencia del dispositivo, lo que permite una gran acogida en dicha población. El robot publicitario posee diversas funciones: la función baile implementada en el robot estimula la coordinación en los movimientos de los niños al ejecutar movimientos rítmicos al mismo tiempo que estimula la memoria infantil y las neuronas espejo (Hickock, 2015), mientras observan o en el mejor de los casos mientras imita al robot; al estimular la memoria y la coordinación motriz en los niños se estimula el desarrollo neurológico de dichos procesos.

Durante la infancia el neurodesarrollo requiere de la adecuada estimulación de las áreas corticales, que permiten los procesos de aprendizaje iniciando desde los más primitivos hasta la maduración de las funciones ejecutivas sirviéndose de la plasticidad cerebral, el ambiente provee de los estímulos extrínsecos para el proceso de sinaptogénesis (Korzeniowski, 2011). En el proceso de descubrimiento del entorno acorde a las demandas individuales, el acceso a medios interactivos supone un incremento en la gama de estímulos al alcance de las habilidades de los niños.

Asi, la atención que mantenga un infante depende de la calidad y duración de los estímulos que excitan el sistema nervioso al ser no solo receptados sino percibidos a nivel consiente, en este ámbito las Tic's forman parte de una herramienta diferente para transmitir información al ser orientadas a la educación combinando estímulos auditivos, visuales y motrices a la diversidad de opciones que permite una unidad robótica interactiva (Kim \& Jong-Hwan, 2004). Presentando una estética infantilizada el aparato electrónico capta el interés y la curiosidad en un ambiente amigable para el infante, predisponiendo el deseo a manipular las diversas opciones propuestas en este robot, como proyección de videos, captura de imágenes, reproducción de cuentos con imitaciones de lenguaje articulado, movimiento rítmico (Víctor, Riveros, \& Mendoza, 2005).

La opción, selección de funciones situada en la pantalla inferior estimula la coordinación mano-ojo requerida al enfocar y tomar objetos, la planeación, ejecución e inhibición de actividades y pensamientos relacionada a las funciones ejecutivas son requeridas para el manejo de este dispositivo siendo la corteza frontal y pre-frontal los encargados del proceso (Franco \& Sousa, 2011).

La función de reproducir cuentos estimula el lóbulo temporo-parietal y prefrontal vinculados con el lenguaje verbal, no verbal y comprensivo, además, la proyección de imágenes mantiene el interés y estimula las áreas relacionadas al procesamiento de imágenes en la corteza parieto-occipital vinculados al reconocimiento facial y pareo de imágenes (Coelho, Fernandes, 
Ribeiro, \& M., 2006). El carácter emocional provisto al relato reproducido con la voz de uno de los padres activará el sistema límbico y la corteza cerebral y subsistemas físicos generando una experiencia afectiva, motivadora de comportamiento (Lizeretti, Gimeno-Bayón, \& Cobos, 2014), resultando un complemento en la formación del vínculo diádico cuando no existe contacto directo con los padres (Botero, 2012).

Motivando la interacción y el placer de la realización de actividades interactivas con el dispositivo electrónico vinculado al aprendizaje de vocabulario de lenguas como el quichua, inglés y español en la infancia incrementa la pronunciación correcta de segundas lenguas (Lenneberg, 2016). El bucle fonológico vinculado a la memoria de trabajo funciona como canal de entrada para el lenguaje subvocal.

La imitación es un canal de aprendizaje por repetición, sin embargo las neuronas espejo realizan una imitación mental, activan las áreas de la circunvolución frontal inferior y la corteza parietal inferior pero no necesariamente las áreas motoras del cerebro. El modo baile de dispositivo se mueve al ritmo de música preseleccionada otorgando estímulos visuales que excite las áreas del sistema de imitación (Rizzolatti, 2006).

\section{Pruebas}

Las pruebas de desplazamiento del robot se realizaron en superficies con un ángulo de inclinación de $0^{\circ}$ respecto a la superficie horizontal y con un índice de fricción cinético de $0.5 \mu \_\mathrm{k}$ que corresponde a la fricción entre el material de las ruedas que es polímero sintético (hule) y el concreto sólido.

Las primeras realizadas fueron el envío de órdenes aleatorias a los brazos y las piernas, logrando como respuesta la realización de dichos movimientos de manera satisfactoria, así como la realización de movimientos de avance, retroceso, giros y traslación, cumpliendo con la velocidad programada en los tiempos determinados por el usuario. Los resultados de las pruebas indican que se obtuvieron tiempos de respuesta rápidos en el accionamiento de los sensores, mismo que se evalúa desde el envío de la orden desde la tarjeta de la tarjeta de control hasta que se obtiene el movimiento en el actuador seleccionado.

Las pruebas para evaluar la comunicación entre el master Raspberry y el esclavo Arduino consistieron en el envío de comandos desde el serial del Arduino y se verificó su recepción en el GPIO I2C. Se realizó una prueba de aceptación del robot al usuario, con una encuesta a los usuarios que tuvieron la oportunidad de interactuar con el robot; y la respuesta de los asistentes demostraron una aceptación favorable, dicha aceptación se puede también visualizar en la página de la red social Facebook del Robot, la misma que permite observar la acogida del robot por parte de los usuarios, Figura 10. 


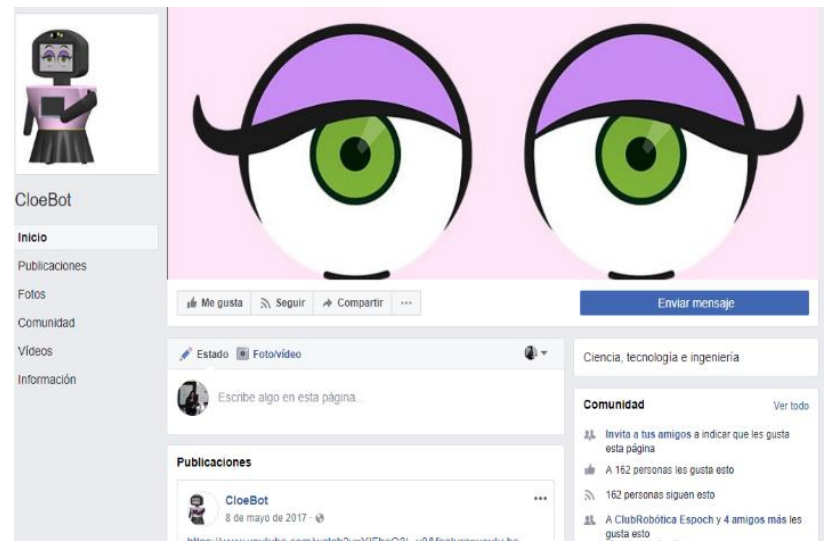

Figura 10. Página de Red social del robot Fuente: Vaca I, Villagómez L.

\section{Conclusion}

Las funciones baile, cuento, y vocabulario que tiene implementado el robot, aportan al desarrollo psicomotriz de los niños. Al transmitir información al usuario mediante la función publicidad del robot se obtienen un nivel de impacto superior al que se obtiene con la trasmisión de información a través de carteles, pantallas, trípticos, vallas publicitarias, entre otros medios convencionales de publicidad. El grado de interacción que presenta el robot ante el usuario permite crear un ambiente familiar lo que estimula el correcto desarrollo del funcionamiento del robot, logrando obtener de ésta manera el resultado esperado, y evidenciando la fácil adaptación del usuario ante el funcionamiento del robot. La aceptación del usuario se observa en la página web de la red social implementada.

Como mejoras futuras del robot se puede trabajar en el procesamiento de las imágenes que se obtienen con la cámara web implementada, la inserción de nuevas actividades lúdicas, así como herramienta en terapias de lenguaje orientadas a los niños con dislalia, lenguaje farfullante o un retraso de la adquisición del lenguaje entre otros, y obtener retro alimentación correspondiente de la eficacia de dichas terapias. Por su parte la estabilidad del robot puede ser mejorada con la implementación de un PID, para los motores de la base del robot.

\section{References:}

1. Acosta, A. (2010). El Buen Vivir en el camino del post-desarrollo. Una lectura desde la Constitución de Montecristi, (págs. 1-36). Policy paper.

2. Aracil, R., Balaguer, C., \& Armada, M. (2008). Robots de servicio. Revista Iberoamericana de Automática e Informática Industrial RIAI, 6-13. 
3. Botero, H. (2012). Relación Madre-hijo. . En El Amor en el Desarrollo del Cerebro del Bebé - Separación Temprana - Patrón de Relaciones y Salud Mental (págs. 133-175). DialnetNeuropsicologiaInfantilDelDesarrollo .

4. Coelho, A., Fernandes, C., Ribeiro, C., \& Victoria, M. (2006). El modelo de Alexander Romanovich Luria (revisitado) y su aplicación a la evaluación neuropsicológica. Revista Galego-portuguesa de psicoloxía e educación, 13, 11-12. issn: 1138-1663.

5. Cortés Cherta, M. (1995). Curso moderno de máquinas eléctricas rotativas. ISBN: 978-84-7146-053-0. Barcelona : Reverté S. A.

6. Diaz, R. (2016). Comunicación entre Raspberry PI y Arduino por USB. rdiaz.es.

7. Franco, J., \& Sousa, L. (2011). Lóbulos frontales y funciones ejecutivas. Revista del Hospital.

8. González, V. (2002). Control automatizado y robótica.

9. Hickock, G. (2015). THE MYTH OF MIRROR NEURONS. THE REAL NEUROSCIENCE OF COMMUNICATION AND COGNITION., ISBN: 978-1-63057-003-3.

10. Ibarra Quevedo, R., \& Arteaga Bouchan, M. G. (2007). Un Ambiente de Aprendizaje con la Robotica Pedagógica para Embalaje.

11. Jyh-Hwa, L., T. Y., \& Kuo, S. (2008). The Development of the Restaurant Service Mobile Robot. In Control Conference, 2008. CCC 2008. (págs. 662-666). IEEE.

12. Kim, \& Jong-Hwan (2004). Soccer Robotics. Springer Science \& Business Media, ISBN: 978-3-540-21859-3.

13. Korzeniowski, C. (2011). Desarrollo evolutivo del funcionamiento ejecutivo y su relación con el aprendizaje escolar. Revista de Psicología., 7-26.

14. Lenneberg, E. (2016). The Biological Foundations of Language. Hospital Practice Lenneberg., 59-67.

15. Lizeretti, N., Gimeno-Bayón, A., \& Cobos, A. (2014). Inteligencia emocional y valores éticos. Revista de psicoterapia., 25, 79-104.

16. Pedraza, O. (2014). Impacto Social Y Académico De La Implementación De Proyectos Roboticos En Colegios Públicos En Bogotá.

17. Raspberry, F. (2016). Raspberry Pi Foundation Uk.

18. Rizzolatti, G. (Abril/Mayo de 2006). Neuronas Espejo. Revista Scientific American Mind, 17.

19. Sanz, S. (2011). Vehículos híbridos y eléctricos (Motores). s.l. Editex ISBN: 978-84-9003-195-7. 
20. Silva Ortigoza, R. \&., Molina Vilchis, M., Hernández Guzmán, V., \& Silva Ortigoza, G. (2007). Una panorámica de los robots móviles. Télématique, , 1-14.

21. Somolinos Sánchez, J. A. (2002). Avances en robótica y visión por computador. Univ de Castilla La Mancha. ISBN: 978-84-8427-199-4.

22. Vega, J., \& López, J. (2006). Oficial de Mantenimiento. ISBN: 97884-665-5181-6. España: MAD-Eduforma.

23. Víctor, S., Riveros, V., \& Mendoza, M. (Septiembre de 2005). Bases Teoricas para el uso de las tics en educación. Encuentro Educacional., 12(3), 315 - 336.

24. Wheat, D. (2012). Arduino Internals. s.l. s.l. : Apress. ISBN: 978-14302-3883-6.

25. Zabala, G. (2007). Robótica. USERSHOP. ISBN: 978-987-1347-56-8, 18. 